\title{
Empatía y juicios morales en población anciana
}

\author{
Helga Ortega, Raúl Cacho, José J. López-Goñi, Javier Tirapu-Ustárroz
}

Introducción. La cognición social se refiere a los procesos mentales que operan en situaciones de interacción social y facilitan el ajuste y el funcionamiento en tales escenarios.

Objetivo. Estudiar la respuesta empática en dos grupos de personas mayores y su relación con la inteligencia emocional y el juicio moral.

Sujetos y métodos. Participaron 60 sujetos divididos en dos grupos de 30 cada uno, que cumplimentaron una batería de pruebas: Trait Meta-Mood Scale-24, cuestionario disejecutivo, índice de reactividad interpersonal (IRI) y dilemas morales.

Resultados. En la dimensión de toma de perspectiva del IRI, el grupo de edad avanzada puntuó significativamente menos que el grupo de mediana edad $(U=279 ; p<0,05)$. En el resto de variables no se encontraron diferencias estadísticamente significativas.

Conclusiones. Los resultados muestran la ausencia de un déficit generalizado en la cognición social en la muestra de ancianos evaluada. Sin embargo, se aprecian diferencias en función de la edad en la empatía y en el rendimiento ejecutivo: con el paso del tiempo tiene lugar un deterioro progresivo en la teoría de la mente y un declive en la capacidad empática general. Con respecto a la inteligencia emocional, los ancianos evaluados manifiestan una adecuada percepción y comprensión de sus emociones, aunque informan de una peor capacidad para manejar y regular sus afectos.

Palabras clave. Empatía. Envejecimiento. Inteligencia emocional. Juicio moral. Teoría de la mente.

\section{Introducción}

El individuo, en las relaciones que establece con otros, requiere no sólo tener conocimientos sobre sus propios deseos e intenciones, sino también ser capaz de inferir las expectativas, creencias y necesidades de su interlocutor, teniendo en cuenta, además, el contexto social en que se desarrollan para que estas relaciones sean exitosas [1-3]. Estos procesos y mecanismos mentales se engloban en lo que se ha denominado cognición social $[4,5]$. La cognición social se refiere a los procesos mentales que operan en situaciones de interacción social y facilitan el ajuste y el funcionamiento en tales escenarios. Comprende aspectos como el procesamiento emocional, la percepción y el conocimiento social, la teoría de la mente (ToM), la empatía y el juicio moral [6].

Muchos de estos aspectos se han estudiado en diferentes poblaciones de individuos. Por ejemplo, se ha estudiado la empatía en niños y adolescentes [7], el juicio moral en adultos sanos [8], el procesamiento de las expresiones faciales en función de la edad [9], el reconocimiento de las expresiones faciales y la ToM en pacientes con demencia [10], la ToM en pacientes con esquizofrenia [11] o el juicio moral en sujetos con daño cerebral [12]. Estas in- vestigaciones han puesto de manifiesto la implicación de diferentes áreas cerebrales en los distintos aspectos de la cognición social. Así, por ejemplo, estudios de neuroimagen revelan la activación de un sistema neural integrado por áreas de la corteza prefrontal medial, el polo temporal y el surco temporal superior en tareas que implican ToM $[13,14]$. Otras áreas cerebrales, como la corteza prefrontal medial y dorsolateral, el cingulado posterior, el surco temporal superior, la amígdala y el lóbulo parietal inferior bilateral, se han implicado en los distintos procesos que subyacen a la conducta y el juicio moral [8,15-17]. También estudios de neuroimagen han puesto de manifiesto dos sistemas de empatía -anatómica y funcionalmente- diferenciados, aunque interrelacionados: un sistema de corte más emocional y filogenéticamente más antiguo [18], relacionado con el contagio emocional que implicaría estructuras límbicas como la amígdala, la corteza somatosensorial derecha, el polo temporal derecho, la corteza cingulada anterior y la ínsula [19-22]; y otro sistema con una respuesta empática más evolucionada [18], de tipo cognitivo ('comprendo lo que tú sientes'), que se encuentra estrechamente vinculada con la ToM y que activa regiones cerebrales como la corteza prefrontal ventromedial, el surco
Departamento de Psicología; Residencia Ntra. Sra. de Gracia (H. Ortega). Departamento de Psicología y Pedagogía; Universidad Pública de Navarra (R. Cacho, J.J. López-Goñi). Servicio de Neuropsicología y Neuropsiquiatría; Clínica Ubarmin (J. Tirapu-Ustárroz). Pamplona, Navarra, España.

Correspondencia:

Dra. Helga Ortega. Departamento de Psicología. Residencia Ntra. Sra. de Gracia. Pl. Constitución, s/n.

E-31500 Tudela (Navarra).

E-mail:

helgaortega@gmail.com

Aceptado tras revisión externa: 23.05.14.

Cómo citar este artículo: Ortega H, Cacho R, López-Goñi JJ, Tirapu-Ustárroz J. Empatía y juicios morales en población anciana. Rev Neurol 2014; 59: 97-105.

(c) 2014 Revista de Neurología 
temporal superior, la unión temporoparietal y el polo temporal [23-26].

En definitiva, cuando se han estudiado las diferentes dimensiones de la cognición social, se ha encontrado una amplia variedad de sistemas cerebrales diferentes, pero relacionados entre sí, que han puesto al descubierto las alteraciones de una amplia variedad de patologías.

Por otro lado, es bien conocida la existencia de un declive cognitivo con la edad y es lógico pensar que este declive también puede ocurrir en las diferentes dimensiones de la cognición social. Sin embargo, a pesar de la amplia investigación sobre alteraciones cognitivas en la vejez y durante el proceso de envejecimiento, se ha prestado menos atención a la cognición social en esta etapa de la vida y su posible influencia en las relaciones que los ancianos establecen con su entorno. El cuerpo de investigaciones sobre cognición social en individuos ancianos se ha centrado principalmente en el procesamiento emocional, entendido como la habilidad para entender, expresar y manejar las emociones y el reconocimiento facial de las expresiones. La conclusión general es que los ancianos muestran mayores dificultades para comprender las expresiones faciales emocionales y, más concretamente, las relativas a la tristeza, la ira y el miedo. Este déficit se ha relacionado con la mayor afectación que tiene lugar en regiones frontales y temporales durante la senectud. Además, dicha dificultad parece ser independiente de la afectación de otras funciones cognitivas, como las habilidades visuoperceptivas, la velocidad de procesamiento, la memoria de trabajo $\mathrm{u}$ otras capacidades [27-29].

Un aspecto muy poco estudiado en ancianos sin patología cognitiva son los juicios y la conducta moral. Estos aspectos se han valorado principalmente empleando los dilemas morales elaborados por Greene et al [8]. Estos autores clasificaron los dilemas morales en dos categorías: 'personales' e 'impersonales'. La primera categoría implica valorar situaciones en que se debe actuar sobre un ser animado, como un ser humano. Por ejemplo, empujar a una persona desde un puente para detener un vagón de tren fuera de control que va a matar a cinco personas. La segunda categoría (dilemas impersonales) se presenta cuando el sujeto aplica la acción sobre un ser inanimado o se realizan violaciones de las reglas sociales. Por ejemplo, apretar un interruptor para desviar un vagón de tren que está fuera de control para salvar a cinco personas que están en las vías, pero que a cambio matará a una persona.

La empatía es un aspecto más estudiado en los ancianos, ya que desempeña un papel crucial en las interacciones sociales en general, en los juicios morales en particular y está relacionada con la ToM. La respuesta empática afecta tanto los aspectos 'fisiológicos' como los 'cognitivos', ya que no sólo activa y depende de estados emocionales propios, sino que implica, además, entender y valorar cómo se siente el otro, 'ponerse en su lugar', de manera que el juicio moral resultante sea acorde con los motivos o intereses no sólo del sujeto en cuestión, sino del colectivo afectado por esa decisión. Así, tanto la empatía emocional como la cognitiva son requisitos esenciales para un funcionamiento social satisfactorio [30]. Algunos estudios recientes han sugerido que la empatía cognitiva, pero no la afectiva, puede deteriorarse como consecuencia del envejecimiento. Un estudio de López-Pérez y Fernández-Pinto [31], que comparó cinco grupos diferentes de edad -adolescencia (16-19 años), adultos jóvenes (20-30 años), edad adulta media (31-40 años), edad adulta tardía (41-59 años) y vejez (60-66 años)-, encontró que mientras los componentes afectivos de la empatía tienden a permanecer estables, los componentes cognitivos tienden a disminuir con la edad. Bailey et al [30] encontraron datos similares comparando dos grupos de adultos jóvenes y adultos mayores. Mientras que con respecto a la empatía emocional no observaron diferencias entre grupos, encontraron una relación negativa entre la edad y la empatía cognitiva. Además, estos mismos autores descubrieron una disminución de las relaciones sociales en los adultos mayores relacionada parcialmente con la disminución en la empatía cognitiva, lo que indica que este declive cognitivo es especialmente importante con respecto a los ancianos, que son particularmente susceptibles a las consecuencias físicas y mentales negativas de la soledad y el aislamiento social [30]. Sin embargo, un reciente estudio de Hühnel et al [3], en el que un grupo de jóvenes y un grupo de ancianos tenían que evaluar diferentes expresiones faciales espontáneas, sólo encontró un deterioro parcial de la empatía cognitiva. Además, otro estudio de Chen et al [2], en el que mediante imágenes de resonancia magnética funcional se evaluaron las respuestas neurales de tres grupos de edad durante el visionado de diferentes imágenes dolorosas, observó una disminución de la empatía emocional relacionada con la edad y no encontró diferencias entre los grupos con respecto a la empatía cognitiva.

Por lo tanto, las reacciones empáticas de los ancianos pueden no estar tan afectadas como algunos estudios sugieren, o pueden estar mediadas por otros procesos cognitivos y emocionales. En consecuencia, los objetivos del presente trabajo son estudiar la respuesta empática en dos grupos de perso- 
nas mayores y su relación con la inteligencia emocional y el juicio moral. Se parte del supuesto de que, en ausencia de enfermedad neurodegenerativa, las modificaciones cerebrales producto de la edad avanzada darán lugar a un detrimento en la capacidad para empatizar con los sentimientos ajenos. Esto implicará una mayor tasa de aprobación de dilemas morales impersonales y personales, en comparación con adultos de menor edad. Asimismo, se supone que un patrón normal de envejecimiento cerebral implicará que la vertiente cognitiva de la empatía se verá afectada siguiendo una progresión lineal ascendente. Es decir, que los adultos muy mayores (80-90 años) tendrán más dificultades con la comprensión empática de los sentimientos ajenos que los adultos mayores (60-70 años), lo que se reflejará en una diferencia entre las pruebas aplicadas en la presente investigación.

\section{Sujetos y métodos}

\section{Participantes}

En este estudio participaron 60 sujetos divididos en dos grupos de 30 sujetos cada uno. El grupo experimental se formó con personas de edad avanzada (media: 75,5 \pm 8,9 años). De ellos, 22 eran mujeres $(73,3 \%)$ y ocho eran hombres $(26,7 \%)$. El grupo de control se conformó con sujetos de mediana edad (media: 43,5 \pm 8,8 años). De ellos, 19 eran mujeres $(63,3 \%)$ y 11 eran hombres $(36,7 \%)$.

Los criterios de inclusión fueron aceptar la participación en el estudio y no cumplir ningún criterio de exclusión. A su vez, los criterios de exclusión fueron los siguientes: tener o haber tenido historia de daño neurológico, tener o haber tenido algún trastorno psicopatológico, padecer algún tipo de deterioro cognitivo o tomar alguna medicación psicofarmacológica.

Todos los participantes dieron su consentimiento informado por escrito para participar en el estudio, y la investigación se condujo según la declaración de Helsinki.

\section{Instrumentos}

\section{Trait Meta-Mood Scale-24 (TMMS-24) [32],} versión española de Fernández-Berrocal et al [33] Esta escala evalúa las habilidades de inteligencia emocional a través de tres dimensiones claves, con ocho ítems cada una de ellas:

- Percepción emocional: capacidad de sentir y expresar los sentimientos de forma adecuada.
- Comprensión de sentimientos: capacidad de comprender adecuadamente los estados emocionales propios.

- Regulación emocional: capacidad de regular los estados emocionales correctamente.

Las puntuaciones obtenidas en cada uno de los factores presentan puntos de corte diferenciados según sexo, pues existen diferencias en las puntuaciones para cada uno de ellos. La versión española ha demostrado una alta consistencia interna y fiabilidad test-retest, de 0,9 (percepción), 0,9 (comprensión) y 0,86 (regulación). Además, con el fin de obtener una medida general de la inteligencia emocional, se creó una variable denominada 'inteligencia emocional general', usando la media conjunta de las puntuaciones de las tres subescalas del TMMS-24.

\section{Cuestionario disejecutivo (DEX-Sp) [34]}

Este cuestionario forma parte de la batería Behavioural Assessment of the Dysexecutive Syndrome [35]. El DEX es un buen instrumento de cribado de las funciones ejecutivas por sus buenas propiedades psicométricas $[34,36]$. Es un cuestionario de 20 ítems puntuado según una escala tipo Likert de 1 ('nunca') a 5 ('con mucha frecuencia'). Tiene dos versiones: una cumplimentada por el propio sujeto (el DEX) y otra que cumplimenta alguna persona de su entorno. En este trabajo se utilizó la versión autoinformada. El DEX se ha aplicado en el estudio de diferentes poblaciones clínicas $[37,38]$, así como en población no clínica $[39,40]$.

\section{Índice de reactividad interpersonal (IRI) [41], en su versión española [42]}

Instrumento ampliamente utilizado que evalúa la empatía desde una perspectiva multidimensional. Consta de 28 ítems que se agrupan en cuatro subescalas:

- Toma de perspectiva: valora la tendencia del sujeto para adoptar, de manera espontánea, el punto de vista del otro.

- Fantasía: mide la predisposición a identificarse con personajes de ficción (en novelas, películas, juegos, etc.).

- Empatía: mide los sentimientos de compasión y preocupación por otros.

- Aflicción personal: se refiere al malestar y la incomodidad generada en el individuo ante la observación de experiencias negativas que suceden a otras personas.

El IRI ha demostrado buenas propiedades psicométricas [42]. Las dos primeras subescalas (toma de perspectiva y fantasía) valoran la empatía cognitiva, 
relacionada con respuestas más evolucionadas y de alta cognición, mientras que las escalas empatía y aflicción personal evalúan la empatía emocional, relacionada con respuestas de contagio emocional más automáticas y primarias [23,43]. Por este motivo se crearon dos variables denominadas 'empatía cognitiva general' y 'empatía emocional general', utilizando la media de las puntuaciones en las subescalas toma de perspectiva y fantasía para la empatía cognitiva general, y empatía y aflicción personal para la empatía emocional general.

\section{Dilemas morales [8]}

Valora la influencia de las emociones, expresadas a través de las respuestas empáticas, y la razón, representada por los procesos cognitivos de alto nivel, en el establecimiento de un juicio moral. Para ello plantea 20 historias cortas, de contenido ético, donde se describe una situación hipotética y se ofrece una solución. Los sujetos deben decidir si aprueban o no la propuesta ofrecida en el dilema. Se dividen en dos grupos de 10 dilemas cada uno:

- Dilemas impersonales: la acción propuesta se aplica sobre un ser inanimado o se requiere violar una regla, de manera que activa una respuesta emocional mínima.

- Dilemas personales: la acción se dirige hacia un ser vivo, por lo que conlleva una mayor implicación emocional para su resolución.

Se utilizó la suma total de respuestas que los sujetos indicaron como adecuadas tanto en los dilemas personales como en los dilemas impersonales.

\section{Procedimiento}

Los participantes fueron evaluados por una psicóloga clínica entrenada en la aplicación de los instrumentos de evaluación. Las sesiones fueron individuales, con una duración aproximada de 60 minutos. Los sujetos cumplimentaron las escalas descritas en el orden expuesto.

\section{Análisis de datos}

Se realizaron análisis descriptivos y de frecuencias para describir las características de los participantes, y análisis no paramétricos (prueba $U$ de MannWhitney para dos muestras independientes y prueba de $\chi^{2}$ ) para analizar las diferencias entre ambos grupos en las diferentes pruebas. Para observar las posibles relaciones entre variables se realizaron análisis de correlaciones usando el coeficiente de correlación de Pearson. En todos se consideró signifi- cativa una $p<0,05$. Todos los análisis estadísticos se realizaron con el programa SPSS v. 15.0.

\section{Resultados}

\section{Comparación entre grupos}

En la tabla I se muestra comparación en las variables clínicas entre los grupos. En la dimensión de toma de perspectiva del IRI, el grupo de edad avanzada puntuó significativamente menos que el grupo de mediana edad $(U=279 ; p<0,05)$. En el resto de variables no se hallaron diferencias estadísticamente significativas.

\section{Relación entre dimensiones del grupo experimental}

La tabla II muestra las correlaciones entre las dimensiones evaluadas en el grupo de adultos mayores. La edad correlacionó de forma negativa con las puntuaciones obtenidas en las subescalas regulación emocional $(r=-0,395 ; p<0,05)$, toma de perspectiva $(\mathrm{r}=-0,496 ; p<0,01)$ y aflicción personal $(r=-0,397 ; p<0,05)$. También correlacionó de forma negativa con la empatía cognitiva total $(r=$ $-0,497 ; p<0,01)$ y con la empatía emocional total $(r=-0,382 ; p<0,05)$.

La escala total del TMMS-24 correlacionó positivamente con la escala cognitiva total $(r=0,626$; $p<0,01)$ y la subescala toma de perspectiva del IRI $(r=0,514 ; p<0,01)$.

La subescala comprensión correlacionó de forma positiva con la escala cognitiva total del IRI $(r=$ 0,369; $p<0,05)$.

La subescala regulación emocional correlacionó de forma positiva con las subescalas del IRI toma de perspectiva $(r=0,710 ; p<0,01)$ y empatía $(r=0,432$; $p<0,05)$, así como con la escala cognitiva total $(r=$ $0,729 ; p<0,01)$ y la escala emocional total $(r=0,465$; $p<0,01)$.

El DEX correlacionó positivamente con la escala cognitiva total del IRI $(r=0,392 ; p<0,05)$.

La escala cognitiva total del IRI correlacionó con la escala emocional total del IRI $(r=0,514 ; p<0,01)$.

Los dilemas impersonales adecuados correlacionaron de forma positiva con los dilemas personales adecuados $(r=0,644 ; p<0,01)$.

\section{Relación entre dimensiones del grupo control}

En la tabla III se muestran las principales correlaciones entre las diferentes dimensiones en el grupo de adultos jóvenes. La edad correlacionó de manera 
Tabla I. Comparaciones entre los grupos.

\begin{tabular}{|c|c|c|c|c|c|c|c|c|}
\hline & & \multicolumn{2}{|c|}{ Total $(n=60)$} & \multicolumn{2}{|c|}{ Experimental $(n=30)$} & \multicolumn{2}{|c|}{ Control $(n=30)$} & \multirow{2}{*}{$u$} \\
\hline & & Media & $\mathrm{DE}$ & Media & DE & Media & DE & \\
\hline \multicolumn{2}{|c|}{ Cuestionario disejecutivo } & 15,1 & 5,9 & 15,3 & 4,8 & 14,9 & 6,8 & 412 \\
\hline \multirow{4}{*}{$\begin{array}{l}\text { Trait Meta- } \\
\text { Mood Scale-24 }\end{array}$} & Percepción emocional & 22,4 & 6,1 & 22,6 & 5,4 & 22,1 & 6,7 & 418,5 \\
\hline & Comprensión emocional & 26,7 & 5,7 & 26,6 & 4,9 & 26,8 & 6,5 & 444,5 \\
\hline & Regulación emocional & 24,3 & 6,4 & 23,3 & 6,9 & 25,4 & 5,8 & 361 \\
\hline & Inteligencia emocional total & & & 24,1 & 4,2 & 24,7 & 4,5 & \\
\hline \multirow{6}{*}{$\begin{array}{l}\text { Índice de } \\
\text { reactividad } \\
\text { interpersonal }\end{array}$} & Toma de perspectiva & 21,7 & 6,0 & 19,4 & 6,6 & 23,9 & 4,5 & $279^{a}$ \\
\hline & Fantasía & 15,9 & 5,4 & 15,3 & 5,0 & 16,5 & 5,7 & 397,5 \\
\hline & Empatía cognitiva total & 18,8 & 4,2 & 17,3 & 4,1 & 20,1 & 3,9 & $293^{a}$ \\
\hline & Empatía & 26,6 & 4,5 & 25,7 & 4,5 & 27,5 & 4,4 & 340 \\
\hline & Aflicción personal & 17,2 & 3,5 & 17,3 & 3,0 & 17,0 & 4,0 & 416,5 \\
\hline & Empatía emocional total & 21,9 & 2,8 & 21,5 & 2,6 & 22,2 & 3,1 & 406 \\
\hline \multirow{2}{*}{ Dilemas } & Dilemas impersonales acuerdo & 5,7 & 1,9 & 5,6 & 2,0 & 5,8 & 1,8 & 410 \\
\hline & Dilemas personales acuerdo & 2,0 & 1,8 & 2,3 & 2,0 & 1,7 & 1,6 & 340 \\
\hline
\end{tabular}

DE: desviación estándar. a $p<0,05$.

positiva con el DEX $(r=0,45 ; p<0,05)$ y con la empatía emocional total del IRI.

La puntuación total del TMMS-24 correlacionó de forma positiva con la escala cognitiva total $(r=$ $0,475 ; p<0,01)$, con la subescala fantasía $(r=0,382$; $p<0,05)$ y con la subescala empatía del IRI $(r=0,41$; $p<0,05)$.

La subescala del TMMS-24 percepción emocional correlacionó de manera positiva con las subescalas del IRI fantasía $(r=0,398 ; p<0,05)$ y empatía $(r=0,424 ; p<0,05)$, así como con la escala emocional total $(r=0,446 ; p<0,05)$.

La subescala comprensión correlacionó de forma positiva con la escala cognitiva total del IRI $(r=0,396$; $p<0,05)$.

La subescala del TMMS-24 regulación emocional correlacionó de manera positiva con la subescala del IRI toma de perspectiva $(r=0,429 ; p<0,05)$.

El DEX correlacionó positivamente con la empatía emocional total del IRI ( $r=0,399 ; p<0,05)$.

La empatía cognitiva total del IRI correlacionó positivamente con la empatía emocional total $(r=0,455$; $p<0,01)$.
A diferencia del grupo experimental, los dilemas no correlacionaron entre sí.

\section{Discusión}

El objetivo principal del presente trabajo era investigar en qué medida algunos aspectos de la cognición social (la inteligencia emocional, la empatía y el juicio moral) podían verse afectados por la edad y su posible relación entre ellos y el funcionamiento ejecutivo.

Los resultados muestran la ausencia de un déficit generalizado en la cognición social en la muestra de ancianos evaluada. Sin embargo, se aprecian diferencias en función de la edad en la empatía y en el rendimiento ejecutivo. En concreto, los sujetos de mayor edad muestran un deterioro de los aspectos cognitivos de la empatía (no así de los aspectos emocionales). Estos datos coinciden con los de estudios previos que indican que los aspectos cognitivos de la empatía sufren un deterioro relacionado con la edad, mientras que sus aspectos más emo- 
Tabla II. Correlaciones entre dimensiones en el grupo experimental.

\begin{tabular}{|c|c|c|c|c|c|c|c|c|c|c|c|c|c|c|}
\hline & Edad & \multicolumn{4}{|c|}{ Trait Meta-Mood Scale-24 } & DEX & \multicolumn{6}{|c|}{ Índice de reactividad interpersonal } & \multicolumn{2}{|c|}{ Dilemas } \\
\hline \multicolumn{15}{|l|}{ Trait Meta-Mood Scale-24 } \\
\hline Total & $-0,264$ & 1 & $0,662^{b}$ & $0,796^{b}$ & $0,737^{b}$ & 0,098 & $0,626^{b}$ & $0,514^{b}$ & 0,349 & 0,315 & 0,263 & 0,147 & $-0,269$ & $-0,204$ \\
\hline Comprensión (C) & $-0,060$ & $0,796^{b}$ & $0,460^{a}$ & 1 & $0,378^{a}$ & $-0,080$ & $0,369^{a}$ & 0,203 & 0,337 & 0,170 & 0,260 & $-0,099$ & $-0,318$ & $-0,072$ \\
\hline Regulación (R) & $-0,395^{\mathrm{a}}$ & $0,737^{b}$ & 0,094 & $0,378^{a}$ & 1 & 0,074 & $0,729^{b}$ & $0,710^{b}$ & 0,258 & $0,465^{b}$ & $0,432^{a}$ & 0,150 & $-0,282$ & $-0,214$ \\
\hline Cuestionario disejecutivo (DEX) & $-0,229$ & 0,098 & 0,206 & $-0,080$ & 0,074 & 1 & $0,392^{\mathrm{a}}$ & 0,339 & 0,196 & 0,076 & $-0,048$ & 0,203 & $-0,017$ & $-0,155$ \\
\hline Fantasía (Fant) & $-0,161$ & 0,349 & 0,175 & 0,337 & 0,258 & 0,196 & $0,593^{b}$ & $-0,019$ & 1 & $0,387^{\mathrm{a}}$ & 0,297 & 0,219 & $-0,342$ & $-0,204$ \\
\hline Emocional total (E total) & $-0,382^{\mathrm{a}}$ & 0,315 & $-0,014$ & 0,170 & $0,465^{b}$ & 0,076 & $0,514^{b}$ & 0,346 & $0,387^{\mathrm{a}}$ & 1 & $0,815^{b}$ & $0,492^{b}$ & $-0,140$ & 0,026 \\
\hline Empatía (Emp) & $-0,184$ & 0,263 & $-0,175$ & 0,260 & $0,432^{\mathrm{a}}$ & $-0,048$ & $0,450^{a}$ & 0,335 & 0,297 & $0,815^{b}$ & 1 & $-0,103$ & 0,016 & 0,200 \\
\hline Aflicción personal (AP) & $-0,379^{a}$ & 0,147 & 0,239 & $-0,099$ & 0,150 & 0,203 & 0,206 & 0,091 & 0,219 & $0,492^{b}$ & $-0,103$ & 1 & $-0,264$ & $-0,256$ \\
\hline \multicolumn{15}{|l|}{ Dilemas } \\
\hline Impersonales adecuados (IA) & 0,064 & $-0,269$ & 0,023 & $-0,318$ & $-0,282$ & $-0,017$ & $-0,321$ & $-0,140$ & $-0,342$ & $-0,140$ & 0,016 & $-0,264$ & 1 & $0,644^{b}$ \\
\hline Personales adecuados (PA) & 0,267 & $-0,204$ & $-0,136$ & $-0,072$ & $-0,214$ & $-0,155$ & $-0,302$ & $-0,221$ & $-0,204$ & 0,026 & 0,200 & $-0,256$ & $0,644^{b}$ & 1 \\
\hline
\end{tabular}

cionales y automáticos permanecen relativamente estables a lo largo del ciclo vital $[3,30,31]$. Estos datos avalarían la existencia de dos circuitos neurales diferentes -aunque relacionados entre sí- para la empatía $[18,23,44]$.

Como consecuencia de lo anterior, los mayores tienen más dificultades para adoptar el punto de vista de un tercero, lo que supone un deterioro progresivo en la ToM con el paso del tiempo. Este deterioro se ha atribuido a la presencia de deterioro cognitivo [45], al declive natural de otros procesos mentales [46] o incluso a una posible pérdida de integridad de la sustancia blanca [47].

Sin embargo, en este estudio se ha encontrado una relación negativa (en el grupo de mayor edad) tanto en la dimensión cognitiva como en la dimensión afectiva de la empatía. Este dato apunta a un declive de la capacidad empática general a partir de cierta edad.
En cuanto al rendimiento ejecutivo, no se han encontrado diferencias entre los grupos. Este resultado era esperable, ya que uno de los criterios de inclusión en el estudio era no tener deterioro cognitivo evidente. Sin embargo, sí se encontró una relación positiva entre el rendimiento ejecutivo evaluado a través del DEX y la edad, aunque sólo en el grupo de adultos mayores. Una posible explicación es que la prueba utilizada podría ser más sensible a los déficits ejecutivos que ocurren a determinada edad o que los cambios normales en las funciones ejecutivas que se producen en pacientes ancianos podrían ser demasiado sutiles para ser detectados por el DEX. Bennet et al [48] encontraron que el DEX era válido para la detección de síntomas disejecutivos cuando lo cumplimenta un profesional entrenado, y no es así en las versiones autoinformadas o las rellenadas por un familiar. En este estudio se ha empleado la versión autoinformada, por lo que qui- 
Tabla III. Correlaciones entre dimensiones en el grupo de control.

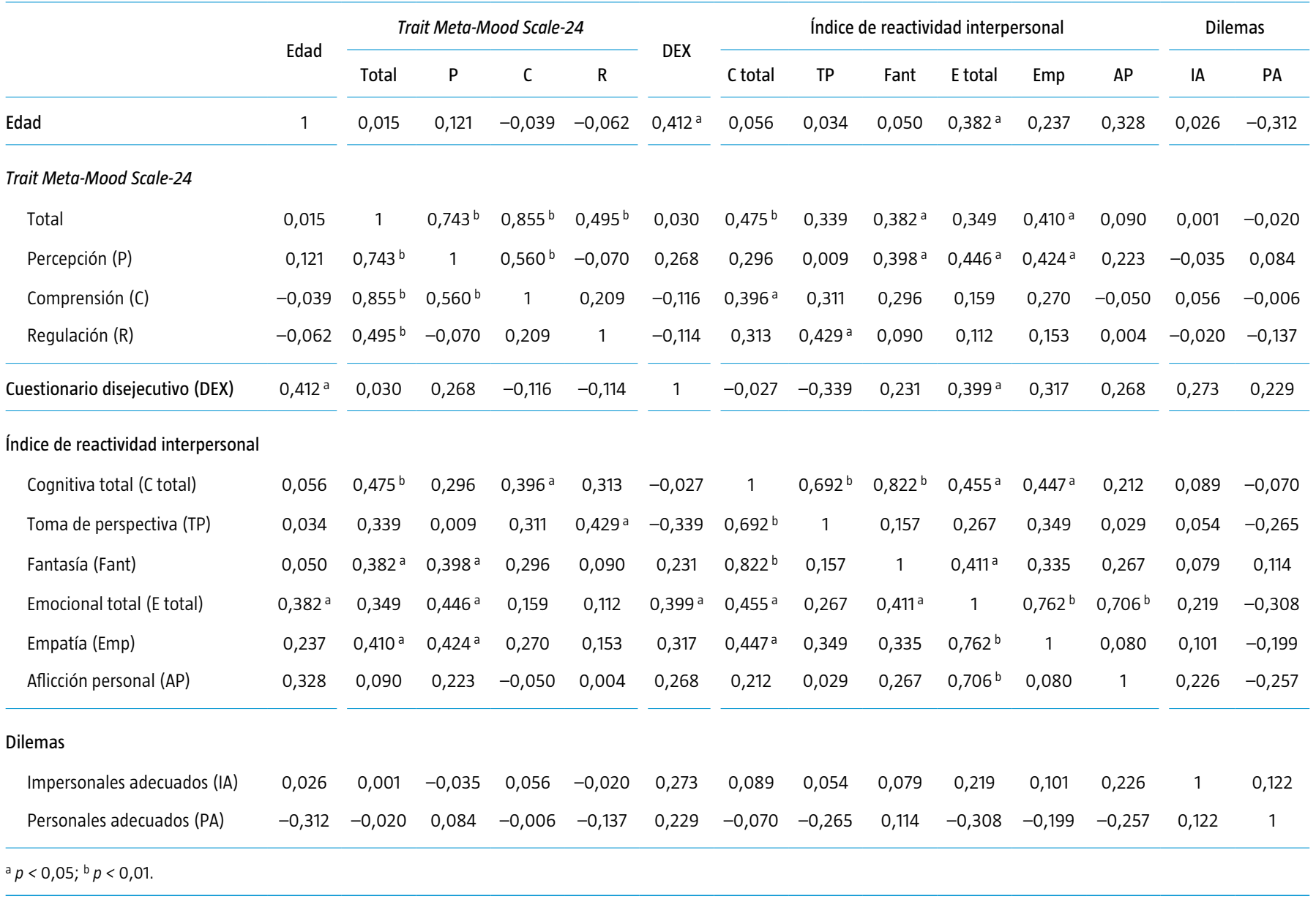

zá no sería sensible a la falta de introspección que pueden tener los adultos muy mayores, de manera que no informan de déficits a ese nivel, aunque sí pueda existir cierto deterioro subyacente en los procesos de inhibición.

Otro dato que apunta a una ausencia de diferencias en los procesos ejecutivos entre los dos grupos son las puntuaciones en los dilemas morales. Estudios de resonancia magnética funcional [8] han confirmado que en los dilemas impersonales se activan las zonas del cerebro más racional, ejecutivo, planificador y evaluativo de riesgos-beneficios. En contraste, en los dilemas personales, la activación se produce en la corteza cingular posterior, la amígdala, la circunvolución temporal superior (surco temporal superior) y la punta del lóbulo temporal [49]. Ambos grupos de sujetos tienden a puntuar como el resto de la población, lo que sugiere que esta capacidad no se ve afectada por el envejeci- miento. Los mayores no tienden a aprobar más soluciones que incurren en violaciones de normas morales ni realizan juicios de carácter utilitarista basados en el bienestar de una mayoría, aunque supongan el sacrificio o infligir un daño directo en una minoría. Tampoco se observa la tendencia contraria, es decir, no muestran unas convicciones morales más rígidas o dogmáticas. Las diferencias entre los datos de este estudio y otros trabajos -como, por ejemplo, el de Moran et al [50]- que sí han encontrado diferencias entre sujetos ancianos y jóvenes en tareas que implicaban juicios morales podrían deberse tanto a las diferencias en las muestras como al uso de medidas distintas. En todo caso, parece que ésta es una interesante línea de investigación para el futuro.

Con respecto a la inteligencia emocional, los ancianos evaluados manifiestan una adecuada percepción y comprensión de sus emociones, similar a la 
del grupo de adultos mayores. Sin embargo, en el grupo experimental, conforme avanza la edad, informan de una peor capacidad para manejar y regular sus afectos. Este dato refuta la hipótesis de la madurez emocional asociada al envejecimiento, que postula que las personas mayores poseen una mayor capacidad para controlar sus emociones, evitando las sobrecargas emocionales, fruto de la experiencia vital acumulada [51].

En este sentido, muchos estudios señalan que los ancianos tienden a emplear mecanismos regulatorios de tipo preventivo, centrados en los antecedentes de la emoción, de manera que seleccionan las relaciones interpersonales y las situaciones en las que se implican, teniendo en cuenta su potencial impacto emocional $[52,53]$. Es posible que en las personas de edad muy avanzada, estas estrategias se tornen ineficaces, bien porque no se aplican de forma apropiada -consecuencia del declive en las funciones mentales que acompaña al envejecimiento- bien porque se ha reducido significativamente el círculo social del anciano y su entorno ya no le provee de la estimulación suficiente, lo que le impide mantener un afecto positivo.

Además, cabe suponer que el mayor control percibido por los mayores no necesariamente supone o garantiza el uso satisfactorio de las estrategias de regulación emocional. Los ancianos pueden recurrir a estrategias preventivas como medio para logar una estabilidad emocional, pero cuando éstas fallan, el empleo de otro tipo de métodos, como la reevaluación cognitiva, la supresión o la rumiación, se tornan ineficaces o, simplemente, no se emplean.

Hay que ser cautos a la hora de generalizar las conclusiones de este estudio. En primer lugar, el tamaño de la muestra es pequeño. Por otra parte, algunos datos se pueden solapar debido a una escasa separación en los rangos de edad entre los grupos. Finalmente, convendría continuar en el futuro con nuevas evaluaciones que incluyan otras variables que influyen en la inteligencia emocional, como los rasgos de personalidad, el índice de satisfacción social y personal, y otras variables neuropsicológicas.

Sin embargo, más allá de estas limitaciones se puede concluir que existe un deterioro, al menos parcial, de la cognición social a medida que avanza la edad, relativamente independiente del resto de funciones cognitivas. Dado que la población anciana es cada vez más numerosa en nuestra sociedad y que demanda más atención, estos datos se deben tener en cuenta a la hora de trabajar y tratar con las personas mayores, y es necesario continuar con la investigación para aclarar las relaciones entre la edad y los aspectos cognitivos y sociales.
Bibliografía

1. Blais C, Roy C, Fiset D, Arguin M, Gosselin F. The eyes are not the window to basic emotions. Neuropsychologia 2012; 50: 2830-8.

2. Chen YC, Chen CC, Decety J, Cheng Y. Aging is associated with changes in the neural circuits underlying empathy. Neurobiol Aging 2014; 35: 827-36.

3. Hühnel I, Fölster M, Werheid K, Hess U. Empathic reactions of younger and older adults: no age related decline in affective responding. J Exp Soc Psychol 2014; 50: 136-43.

4. Adolphs R. Social cognition and the human brain. Trends Cogn Sci 1999; 3: 469-79.

5. Frith CD. Social cognition. Philos T Roy Soc B 2008; 363: 2033-9.

6. Martín-Contero MC, Secades-Villa R, Tirapu-Ustárroz J. Cognición social en adictos a opiáceos. Rev Neurol 2012; 55: 705-12.

7. Schwenck C, Göhle B, Hauf J, Warnke A, Freitag CM, Schneider W. Cognitive and emotional empathy in typically developing children: the influence of age, gender, and intelligence. Eur J Dev Psychol 2014; 11: 63-76.

8. Greene JD, Sommerville RB, Nystrom LE, Darley JM, Cohen JD. An fMRI investigation of emotional engagement in moral judgment. Science 2001; 293: 2105-8.

9. Prodan CI, Orbelo DM, Ross ED. Processing of facial blends of emotion: support for right hemisphere cognitive aging. Cortex 2007; 43: 196-206.

10. Bertoux M, Funkiewiez A, O'Callaghan C, Dubois B, Hornberger $M$. Social cognition and emotional assessment is a marker of medial and orbital frontal functions: a voxel-based morphometry study in behavioral variant of frontotemporal degeneration. J Int Neuropsychol Soc 2012; 18: 972-85.

11. Konstantakopoulos G, Ploumpidis D, Oulis P, Patrikelis P, Nikitopoulou S, Papadimitriou GN, et al. The relationship between insight and theory of mind in schizophrenia. Schizophr Res 2014; 152: 217-22.

12. Koenigs M, Young L, Adolphs R, Tranel D, Cushman F, Hauser M, et al. Damage to the prefrontal cortex increases utilitarian moral judgements. Nature 2007; 446: 908-11.

13. Frith U, Frith, CD. Development and neurophysiology of mentalizing. Philos T Roy Soc B 2003; 358: 459-73.

14. Gallagher HL, Happé F, Brunswick N, Fletcher PC, Frith U, Frith CD. Reading the mind in cartoons and stories: an fMRI study of 'theory of mind' in verbal and nonverbal tasks. Neuropsychologia 2000; 38: 11-21.

15. Mendez MF, Shapira JS. Altered emotional morality in frontotemporal dementia. Cogn Neuropsychiatry 2009; 14: 165-79.

16. Pascual L, Rodrigues P, Gallardo-Pujol, D. How does morality work in the brain? A functional and structural perspective of moral behavior. Front Integr Neurosci 2013; 7: 1-8.

17. Young L, Bechara A, Tranel D, Damasio H, Hauser M, Damasio A. Damage to ventromedial prefrontal cortex impairs judgment of harmful intent. Neuron 2010; 65: 1-7.

18. Decety J, Norman GJ, Berntson GG, Cacioppo JT. A neurobehavioral evolutionary perspective on the mechanisms underlying empathy. Prog Neurobiol 2012; 98: 38-48.

19. Krämer UM, Bahram Mohammadi B, Doñamayor N, Samii A, Münte TF. Emotional and cognitive aspects of empathy and their relation to social cognition -an fMRI-study. Brain Res 2010; 22: 110-20.

20. Völlm BA, Taylor ANW, Richardson P, Corcoran R, Stirling J, McKie S, et al. Neuronal correlates of theory of mind and empathy: a functional magnetic resonance imaging study in a nonverbal task. Neuroimage 2006; 29: 90-8.

21. Rosen HJ, Levenson RW. The emotional brain: combining insights from patients and basic science. Neurocase 2009; 15: 173-81.

22. Hein G, Singer T. I feel how you feel but not always: the empathic brain and its modulation. Curr Opin Neurobiol 2008; 18: 153-8.

23. Shamay-Tsoory SG, Aharon-Peretz J, Perry, D. Two systems for empathy: a double dissociation between emotional and cognitive empathy in inferior frontal gyrus versus ventromedial prefrontal lesions. Brain 2009; 132: 617-27. 
24. Schulte-Ruether M, Markowitsch HJ, Fink GR, Piefke M. Mirror neuron and theory of mind mechanisms involved in face-to-face interactions: a functional magnetic resonance imaging approach to empathy. J Cogn Neurosci 2007; 19: 1354-72.

25. Rizzolatti $\mathrm{G}$. The mirror neuron system and its function in humans. Anat Embryol 2005; 210: 419-21.

26. Moya-Albiol L, Herrero N, Bernal MC. Bases neurales de la empatía. Rev Neurol 2010; 50: 89-100.

27. Sullivan S, Ruffman T. Emotion recognition deficits in the elderly. Int J Neurosci 2004; 114: 403-32.

28. Ryan M, Murray J, Ruffman T. Aging and the perception of emotion: processing vocal expressions alone and with faces. Exp Aging Res 2010; 36: 1-22.

29. Phillips LH, MacLean RDJ, Allen R. Age and the understanding of emotions: neuropsychological and sociocognitive perspectives. J Gerontol B Psychol Sci Soc Sci 2002; 57B: 526-30.

30. Bailey PE, Henry JD, Von Hippel W. Empathy and social functioning in late adulthood. Aging Ment Health 2008; 12: 499-503.

31. López-Pérez B, Fernández-Pinto I. Diferencias de edad en empatía: desde la adolescencia hasta la tercera edad. Ansiedad y Estrés 2010; 16: 139-50.

32. Salovey P, Mayer JD, Goldman SL, Turvey C, Palfai TP. Emotional attention, clarity and repair: exploring emotional intelligence using the Trait Meta-Mood Scale. In Pennebaker JW, ed. Emotion, disclosure and health. Washington DC: American Psychological Association; 1995. p. 125-54.

33. Fernández-Berrocal P, Extremera N, Ramos N. Validity and reliability of the Spanish modified version of the Trait MetaMood Scale. Psychol Rep 2004; 94: 751-5.

34. Pedrero-Pérez EJ, Ruiz-Sánchez de León JM, Olivar-Arroyo A, Bouso-Saiz JC, Rojo-Mota G, Llanero-Luque M, et al. Versión española del cuestionario disejecutivo (DEX-Sp): propiedades psicométricas en adictos y población no clínica. Adicciones 2009; 21: 155-66.

35. Wilson BA, Evans JJ, Alderman N, Burgess PW, Hemslie H. Behavioural of the dysexecutive syndrome. In Rabbit P, ed. Methodology of frontal and executive function. Bury St. Edmunds, UK: Psychology Press; 1996. p. 232-43.

36. Pedrero-Pérez EJ, Ruiz-Sánchez de León JM, Lozoya-Delgado P, Llanero-Luque M, Rojo-Mota G, Puerta-García C. Evaluación de los síntomas prefrontales: propiedades psicométricas y datos normativos del cuestionario disejecutivo (DEX) en una muestra de población española. Rev Neurol 2011; 52: 394-404.

37. Mathias JL. Neurobehavioral functioning of persons with Parkinson's disease. Appl Neuropsychol 2003; 10: 57-68.

38. Walley RM, Donaldson MD. An investigation of executive function abilities in adults with Prader-Willi syndrome. J Intell Disabil Res 2005; 49: 613-25.

39. Mooney B, Walmsley C, McFarland K. Factor analysis of the Self-Report Dysexecutive (DEX-S) Questionnaire. Appl Neuropsychol 2006; 13: 12-8.

40. Wang Y, Chan RCK, Deng YY. Examination of postconcussionlike symptoms in healthy college students: relationships to subjective and objective neuropsychological function performance. Arch Clin Neuropsychol 2006; 21: 339-47.

41. Davis MH. Measuring individual differences in empathy: evidence for a multidimensional approach. J Pers Soc Psychol 1983; 44: 113-26.

42. Pérez-Albéniz A, De Paúl J, Etxeberria J, Montes MP, Torres E. Adaptación del Interpersonal Reactivity Index (IRI) al español. Psicothema 2003; 15: 267-72.

43. De Waal FB. Putting the altruism back into altruism: the evolution of empathy. Annu Rev Psychol 2007; 59: 1-22.

44. Banissy MJ, Kanai R, Walsh V, Reesb G. Inter-individual differences in empathy are reflected in human brain structure. Neuroimage 2012; 62: 2034-9.

45. Pezzuti L, Longobardi E, Milletti K, Ovidi A. A study about the theory of mind in primary and secondary aging. Life Span Disabil 2011; 14: 31-44.

46. Rakoczy H, Harder-Kasten A, Sturm L. The decline in theory of mind in old age is (partly) mediated by developmental changes in domain-general abilities. Br J Psychol 2012; 103: 58-72.

47. Charlton RA, Barrick TR, Markus HS, Morris RG. Theory of mind associations with other cognitive functions and brain imaging in normal aging. Psychol Aging 2009; 24: 338-48.

48. Bennet PC, Ong B, Ponsford J. Measuring executive dysfunction in an acute rehabilitation setting: using the dysexecutive questionnaire (DEX). J Int Neuropsychol Soc 2005; 11: 376-85.

49. Álvaro-González LC. Neuroética (I): circuitos morales en el cerebro normal. Rev Neurol 2014; 58: 225-33.

50. Moran JM, Jolly E, Mitchell JP. Social-cognitive deficits in normal aging. J Neurosci 2012; 32: 5553-61.

51. Lawton MP, Kleban MH, Rajagopal D, Dean J. Dimensions of affective experience in three age groups. Psychol Aging 1992; 7: 171-84.

52. Gross JJ, Carstensen LL, Pasupathi M, Tsai J, Götestam Skorpen C, Hsu AYC. Emotion and aging: experience, expression, and control. Psychol Aging 1997; 12: 590-9.

53. Márquez-González M, Fernández de Trocóniz MI Montorio-Cerrato I, Losada-Baltar A. Experiencia y regulación emocional a lo largo de la etapa adulta del ciclo vital: análisis comparativo en tres grupos de edad. Psicothema 2008; 20 : 616-22.

\section{Empathy and moral judgements in the elderly population}

Introduction. Social cognition refers to mental processes that operate in situations of social interaction and facilitate adjustment and functioning in such scenarios.

Aim. To study the empathic response in two groups of older people and their relationship to emotional intelligence and moral judgment.

Subjects and methods. We enrolled 60 subjects divided into two groups of 30 subjects each completed a battery of tests: TMMS-24, DEX, IRI and moral dilemmas.

Results. In the dimension of the IRI perspective taking older group scored significantly less than the middle age group ( $U=279$; $p<0.05)$. In all other variables, no statistically significant differences were found.

Conclusions. The results show the absence of a generalized deficit in social cognition in the elderly sample evaluated. However, differences were observed based on age on empathy and executive performance: over time a progressive impairment in theory of mind and a decline in the general empathic capacity occur. With regard to emotional intelligence, the elderly evaluated show a correct perception and understanding of their emotions even report a poorer ability to manage and regulate their emotions.

Key words. Aging. Emotional intelligence. Empathy. Moral judgment. Theory of mind. 